\title{
A genetically and functionally diverse group of non-diazotrophic Bradyrhizobium spp. colonizes the root endophytic compartment of Arabidopsis thaliana
}

\author{
Martinus Schneijderberg, Lucas Schmitz, Xu Cheng, Sharon Polman, Carolien Franken, Rene Geurts \\ and Ton Bisseling ${ }^{*}$
}

\begin{abstract}
Background: Diazotrophic Bradyrhizobium spp. are well known for their ability to trigger nodule formation on a variety of legume species. In nodules, Bradyrhizobium utilizes plant-derived carbohydrates in exchange for fixed nitrogen. The genes essential for the nodulation and nitrogen-fixation trait are clustered in a genomic region, which is known as the 'symbiotic island'. Recently, novel non-diazotrophic Bradyrhizobium spp. have been found to be highly abundant in soils, suggesting that these species can also have a 'free-living' life history. However, whether non-diazotrophic Bradyrhizobium spp. can live in association with plants remains elusive.

Results: In this study, we show that Bradyrhizobium spp. are common root endophytes of non-legume plant species - including Arabidopsis thaliana (Arabidopsis) - grown in an ecological setting. From a single Arabidopsis root, four Bradyrhizobium sp. strains (designated MOS001 to MOS004) were isolated. Comparative genome analysis revealed that these strains were genetically and functionally highly diverse, but did not harbour the nodulation and the nitrogen fixation gene clusters. Comparative colonization experiments, with MOS strains and nitrogen-fixing symbiotic strains, revealed that all tested Bradyrhizobium spp. can colonize the root endophytic compartment of Arabidopsis.

Conclusion: This study provides evidence that both diazotrophic and non-diazotrophic Bradyrhizobium spp. colonize the root endophytic compartment of a wide variety of plant species, including the model species Arabidopsis. This demonstrates that plant roots form a major ecological niche for Bradyrhizobium spp., which might be ancestral to the evolution of the nodulation and nitrogen-fixation trait in this genus.
\end{abstract}

Keywords: Bradyrhizobium, Arabidopsis, Root colonization, Endophytic compartment

\section{Background}

Plants can develop relationships with soil bacteria, which vary from loose associations in the rhizosphere up to intimate inter- and intracellular hosting in plant tissues. An eminent and well-studied example of an intimate interaction is the relationship between legumes and a paraphyletic group of nitrogen-fixing $\alpha$ - and $\beta$-proteobacteria, collectively known as rhizobia [1]. Legumes can form special organs on their roots - known as nodules - that

\footnotetext{
* Correspondence: ton.bisseling@wur.nl

Department of Plant Sciences, Laboratory of Molecular Biology, Wageningen University, Droevendaalsesteeg 1, 6708 PB Wageningen, The Netherlands
}

facilitate rhizobia to convert atmospheric nitrogen into ammonium, which subsequently can be utilized by the plant [2]. The bacterial genes underlying this nitrogenfixing nodule symbiosis are the nodulation (nod and nol) and nitrogen fixation (nif and fix) genes. These genes are organized in one or more clusters on the genome or symbiotic plasmid(s). The nod/nol and niflfix genes - as identified in the diverse range of rhizobial genera - are highly homologous and it is therefore widely accepted that these symbiotic genes were transmitted by horizontal gene transfer [3]. This suggests that the lifestyle of ancestral rhizobium species was unrelated to nodule symbiosis. 
One of the most profound clades of symbiotic rhizobia is the genus Bradyrhizobium (within the family of the Bradyrhizobiaceae). Defined as "slow-growing, non-acidproducing root nodule bacteria of leguminous plants" [4], this genus encompasses now 37 species [5]. The type strains of these species all nodulate and have a wide range of legumes (including Glycine max; soybean), and the non-legume Parasponia spp. as host [6, 7]. The genomes of Bradyrhizobium spp. are relatively large (7-10 Mb), and the nodulation (nod/nol) and nitrogen fixation (nif) $f i x)$ gene clusters are located on a symbiotic island within the genome [8].

Recent studies revealed that Bradyrhizobium spp. are also highly abundant in soils in absence of legume plants. At first, evidence came from culture-independent studies on the bacterial communities in soils and rhizospheres of oak trees. These communities harboured large populations of Bradyrhizobium spp. [9-11]. Additional evidence for the abundance of Bradyrhizobium spp. in absence of legumes was found in coniferous forest soils of North America [12]. Through quantitative population genomics and whole-genome sequencing, it was shown that the identified Bradyrhizobium spp. lack the nod/nol and nif/ fix gene clusters, and consequently are incapable to nodulate legumes or to fix atmospheric nitrogen. Instead, these strains possess multiple gene clusters affiliated with complex carbon metabolism and aromatic compound degradation. Likewise, two Bradyrhizobium spp. strains isolated from soils of a bare fallow field and a grassland did not possess the symbiotic island [13]. Together, these studies indicate that Bradyrhizobium spp. can not only live in symbiosis with legumes, but can also have a 'free-living' life history. However, it remains unknown whether such non-nitrogen fixing Bradyrhizobium spp. can have intimate relationships with plants.

As nodulating and nitrogen-fixing Bradyrhizobium spp. can establish an intracellular lifestyle with their legume hosts, we hypothesised that Bradyrhizobium spp. lacking the symbiotic island can live in a close intimate association with plants. In this study, we investigated whether such Bradyrhizobium spp. occur on or inside the roots of nonlegumes in a natural ecosystem. We made use of a dataset from an ecological test field in The Netherlands at the Veluwe area called the Mossel, where we previously studied the root microbiome of nine species, among which Arabidopsis thaliana (Arabidopsis) (Schneijderberg et al., in preparation).

\section{Results}

\section{Bradyrhizobium spp. colonize plant roots}

To determine whether Bradyrhizobium species can colonize non-legume plant roots, we analysed a $16 \mathrm{~S}$ rDNA amplicon dataset from a separate but simultaneous experiment (Schneijderberg et al. in preparation). This dataset comprises a field experiment at the Veluwe area (a region called the Mossel) in The Netherlands and includes nine plant species representing six taxonomic orders (Table 1). Eight of these species are non-legumes, among which is Arabidopsis (accession Mossel; Msl). The bacterial communities of the root endophytic compartments of these species have been determined by $16 \mathrm{~S}$ rDNA amplicon sequencing, after growing for 8 weeks in the summer of 2016 on an experimental plot in the Mossel (Schneijderberg et al. in preparation). Analysis revealed four operational taxonomic units (OTUs) affiliated with the genus Bradyrhizobium, of which one OTU (\#7) was highly abundant in both the soil (0.5\%) and the plant root endophytic compartment (Fig. 1). This OTU was not only abundant in the root endophytic compartment of the legume Lotus corniculatus, but also in root samples of all the eight nonlegumes, ranging from $1.5 \%$ in Leucanthemum vulgare to more than 3\% in Plantago lanceolata (Fig. 1). The widespread abundance of Bradyrhizobium spp. in the root endophytic compartment of all studied plant species indicates that root colonization by strains of this genus is a generic phenomenon in the Mossel area.

To determine whether Bradyrhizobium spp. are root endophytes in other ecosystems as well, we analysed publicly available $16 \mathrm{~S}$ rDNA amplicon datasets. This showed that Bradyrhizobium OTUs are present in the endophytic compartment of a variety of plants, including Arabidopsis, rice (Oryza sativa) and maize (Zea mays) (Table 2). This strongly supports that Bradyrhizobium spp. are common endophytes in plant roots. However, whether the strains that make up the Bradyrhizobium OTUs in those studies possess the symbiotic island, remains elusive.

\section{Arabidopsis endophytic Bradyrhizobium sp. MOS strains lack the nitrogen fixation trait}

An OTU based on the 16S rDNA V4 region can represent multiple strains or even species. To determine whether there are multiple strains belonging to OTU 7

Table 1 Plant species native to the Veluwe Mossel area in the Netherlands that were used in the 165 rDNA meta amplicon analysis

\begin{tabular}{lll}
\hline Species & Order & Common name \\
\hline Arabidopsis thaliana & Brassicales & Thale cress / Arabidopsis \\
Crepis capillaris & Asterales & Smooth hawksbeard \\
Hypericum perforatum & Malpighiales & St John's wort \\
Leucathemum vulgare & Asterales & Ox-eye daisy \\
Lotus corniculatus & Fabales & Bird's-foot trefoil \\
Myosotis arvensis & Boraginales & Field forget-me-not \\
Plantago lanceolata & Lamiales & Narrowleaf plantain \\
Tanacetum vulgare & Asterales & Tansy \\
\hline
\end{tabular}




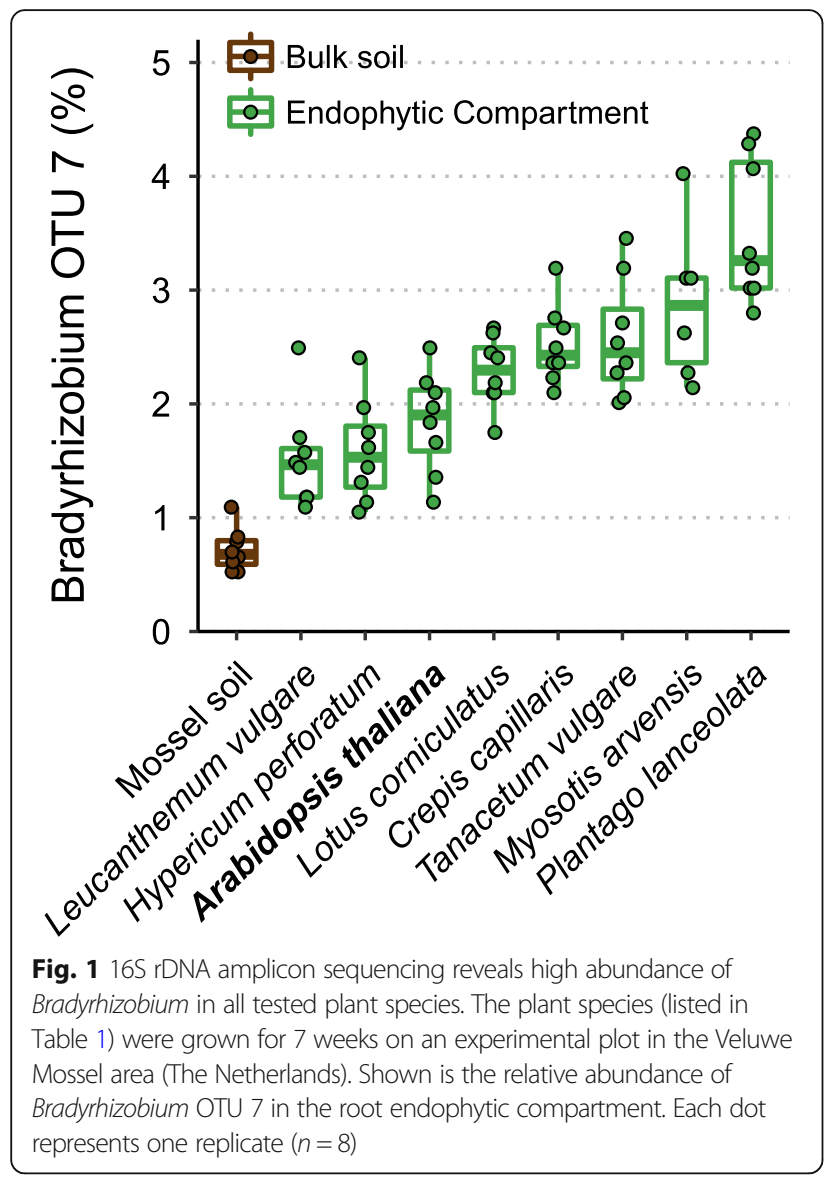

and whether these strains possess the nodulation and nitrogen fixation gene clusters, we set out on an isolation and characterization strategy for Bradyrhizobium spp. From a single Arabidopsis root grown in the Mossel experimental plot, we isolated 102 strains that showed growth characteristics of Bradyrhizobium. These strains were classified in 12 groups based on DNA-fingerprinting using BOX-PCR (Additional file 1). Next, we sequenced the 16S rDNA locus of a representative strain of each group. This revealed four strains (representing $85 \%$ of the isolates) that matched with Bradyrhizobium in the RDP database [14] (Additional file 1). The V4 region of the $16 \mathrm{~S}$ rDNA gene of these four strains showed more than $99 \%$ identity to the consensus sequence of OTU 7, indicating that these isolates represent this OTU. We named these

Table 2 Bradyrhizobium identified in root endophytic compartment

\begin{tabular}{lll}
\hline Species & $\begin{array}{l}\text { Relative } \\
\text { Abundance (\%) }\end{array}$ & Reference \\
\hline Arabidopsis thaliana & 1 & {$[44]$} \\
Arabidopsis thaliana & 3 & {$[45]$} \\
Oryza sativa & 1 & {$[46]$} \\
Zea mays & 0,3 & {$[47]$} \\
\hline
\end{tabular}

strains Bradyrhizobium sp. MOS001 to Bradyrhizobium sp. MOS004, after the area from which they originated. A fifth strain designated MOS005, classified as Bradyrhizobium based on the 16S rDNA gene, was also sequenced. However, since the full genome sequence could not ensure that this was a Bradyrhizobium sp., we chose to exclude it from the analysis (Additional file 1).

To determine whether these Bradyrhizobium spp. MOS strains possess the nitrogen fixation trait, we assembled draft genome sequences based on a 150 base pair paired-end library sequenced on the Illumina HiSeq platform. This resulted in draft assemblies ranging in size from 7.6 (MOS003) to $9.1 \mathrm{Mb}$ (MOS001), representing 6879 (MOS003) to 8622 (MOS001) annotated gene models (Additional file 2). Such genome sizes, as well as the corresponding GC contents (64-66\%), are comparable to previously sequenced Bradyrhizobium spp. genomes $[12,13,15,16]$. To verify the completeness of the draft genome assemblies of Bradyrhizobium sp. MOS strains, Busco analysis was conducted [17]. This revealed full-length presence of $97.6 \%$ in MOS004 and $99.1 \%$ in the other MOS strains of the Rhizobiales test set of 686 genes, indicating close to complete genome coverages (Additional file 2).

To determine whether Bradyrhizobium sp. MOS strains possess nodulation and/or nitrogen fixation genes we used a reciprocal best blast hit algorithm with the nitrogen-fixing symbiont Bradyrhizobium diazoefficiens strain USDA110 as reference [16]. This revealed that the four Bradyrhizobium sp. MOS strains do not have any hit in either the nod/nol gene cluster or the nif/fix gene cluster (Fig. 2). Therefore we conclude that Bradyrhizobium sp. MOS strains are incapable of nodulation and nitrogen fixation.

\section{Arabidopsis endophytic Bradyrhizobium sp. strains are highly diverse}

Based on the genetic fingerprinting the four isolates are different. In order to get insight in the taxonomic diversity represented by the four isolated Bradyrhizobium sp. MOS strains, the phylogenetic relation was determined. Since we obtained full genome sequences, we based our phylogenetic analysis on the nucleotide sequences of 31 AMPHORA genes [18], which comprise a set of highly conserved marker genes. We aligned these genes against all Bradyrhizobium spp. for which genomes have been published, including non-symbiotic strains identified in forest areas (designated LTSP [12]) and grassland (designated G22 and BF49 [13]). Subsequently, the phylogenetic relation was reconstructed (Fig. 3). The topology of the phylogenetic tree is in line with previously reported phylogenies based on single or multiple sequence alignments $[12,19,20]$. The Bradyrhizobium sp. MOS strains represent four distinct lineages within the genus (Fig. 3). 


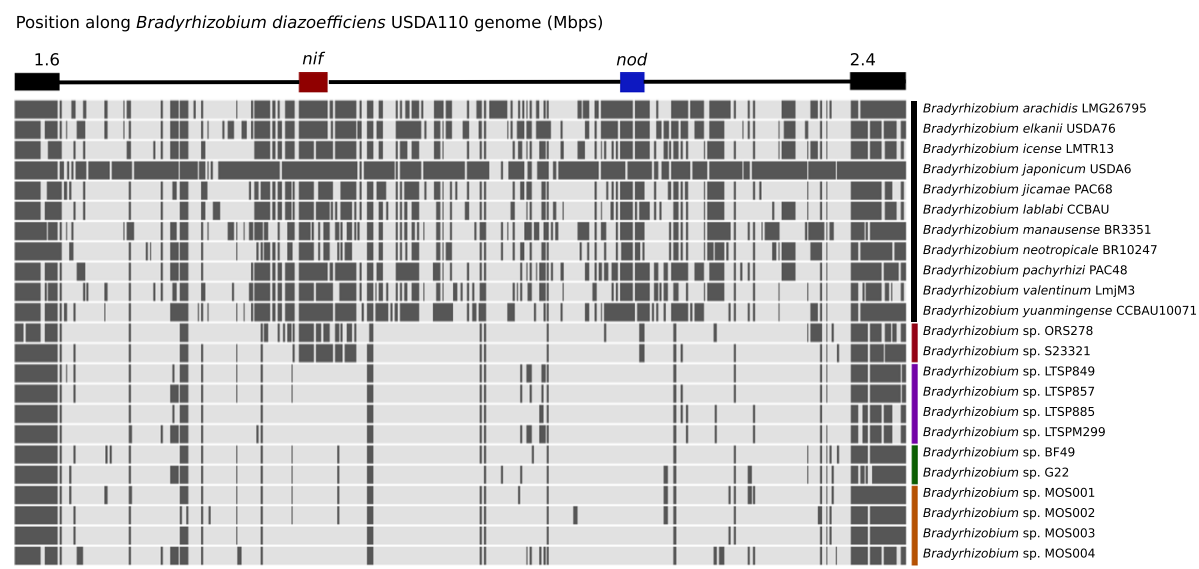

Fig. 2 Reciprocal best blast hit analysis shows that Bradyrhizobium sp. MOS strains lack the symbiotic island. On top a schematic representation of the symbiotic reference strain B. diazoefficiens USDA110, with in black the conserved regions, in red the nif/fix gene cluster and in blue the nod/ nol gene cluster. On the right, the strain name (color-coded as followed. Black: the type strains from Fig. 3; red: strains possessing only the nifffix gene cluster; purple: the non-symbiotic LTSP strains [12]; green: the non-symbiotic strains G22 and BF49 [13]: orange, the MOS strains. Dark grey bar means a hit on the reference genome, with a coverage of $\geq 50 \%$ and a similarity of $\geq 70 \%$. The Bradyrhizobium sp. MOS strains do not have a hit in the nod/nol or nif/fix gene clusters, suggesting absence of the symbiotic island. As expected, the LTSP strains as well as the G22 / BF49 strains show no hits in the symbiotic genes either

Bradyrhizobium sp. MOS001 and MOS002 belong to different lineages of a clade represented by the type strain $B$. japonicum USDA6 and cluster with the two nonsymbiotic strains isolated from grassland (namely Bradyrhizobium sp. G22 and BF49, respectively). Bradyrhizobium sp. MOS003 falls within a large clade, which includes the type strains B. arachidis LMG26795 and B. yuanmingense CCBAU10071, whereas Bradyrhizobium sp. MOS004 shows high sequence homology to Bradyrhizobium sp. DFCI-1, within the B. elkanii USDA76 clade.

Since the four strains originate from a single Arabidopsis root, we questioned whether the isolates are functionally similar, despite their genetic divergence. We used a previously published custom R pipeline [21] to predict the functional groups of all the open reading frames in each of the isolates using the KEGG Orthology (KO) database [22]. After determining presence and absence of the $\mathrm{KO}$ groups and creating a pair-wise dissimilarity matrix using a binary distance measure, we plotted each functional profile along the first two Principal Coordinates. This resulted in a PCoA in which the distances between the species resemble the phylogenetic relationship of the strains (Fig. 4). Along the first two principal coordinates, the Bradyrhizobium sp. MOS001, MOS002 and MOS003 strains cluster relatively close to $B$. diazoefficiens USDA110 and $B$. japonicum USDA6, while MOS004 are relatively close to the B. elkanii clade. This indicates that the group of MOS isolates is not only genetically, but also functionally diverse.

The MOS isolates recolonize the Arabidopsis root We aimed to determine whether Bradyrhizobium sp. MOS001, MOS002, MOS003 and/or MOS004 have the capacity to colonize a plant root endophytic compartment in an experimental system. Furthermore, we questioned to what extent this colonization capacity is similar to the colonization capacity of diazotrophic Bradyrhizobium spp. To investigate this, we set up a growth assay in where 7 days-old in vitro grown Arabidopsis seedlings (Accession Msl) are transplanted into autoclaved river sand inoculated with a Bradyrhizobium strain to a density comparable with the density of Bradyrhizobium in the native Mossel soil, which is approximately $2.5 \times 10^{5}$ cells per gram of soil. After 2 weeks, plants were analysed. None of the four Bradyrhizobium sp. MOS strains, nor any of 3 tested nitrogen-fixing symbiotic strains affected the fresh weight of the Arabidopsis plants (Additional file 3). To quantify root colonization of the different strains, we used qPCR on the highly conserved bacterial $r p o B$ gene and normalized that against tubulin binding co-factor $\mathrm{C}$ of Arabidopsis [23]. While detection of bacterial DNA in the roots of non-inoculated control plants was close to zero, DNA of all strains was detected in the root endophytic compartment samples in the quantity of approximately 2 bacterial genome copies for every plant genome copies (Fig. 5). Comparing the four Bradyrhizobium sp. MOS strains with the three nitrogen-fixing symbionts did not reveal a significant difference in colonization capacity. This shows that symbiotic and non-symbiotic Bradyrhizobium strains colonize the Arabidopsis root equally well in our growth system (Fig. 5).

\section{Discussion}

Here we showed that the endophytic compartment of a variety of non-legume plants - including Arabidopsis - is 


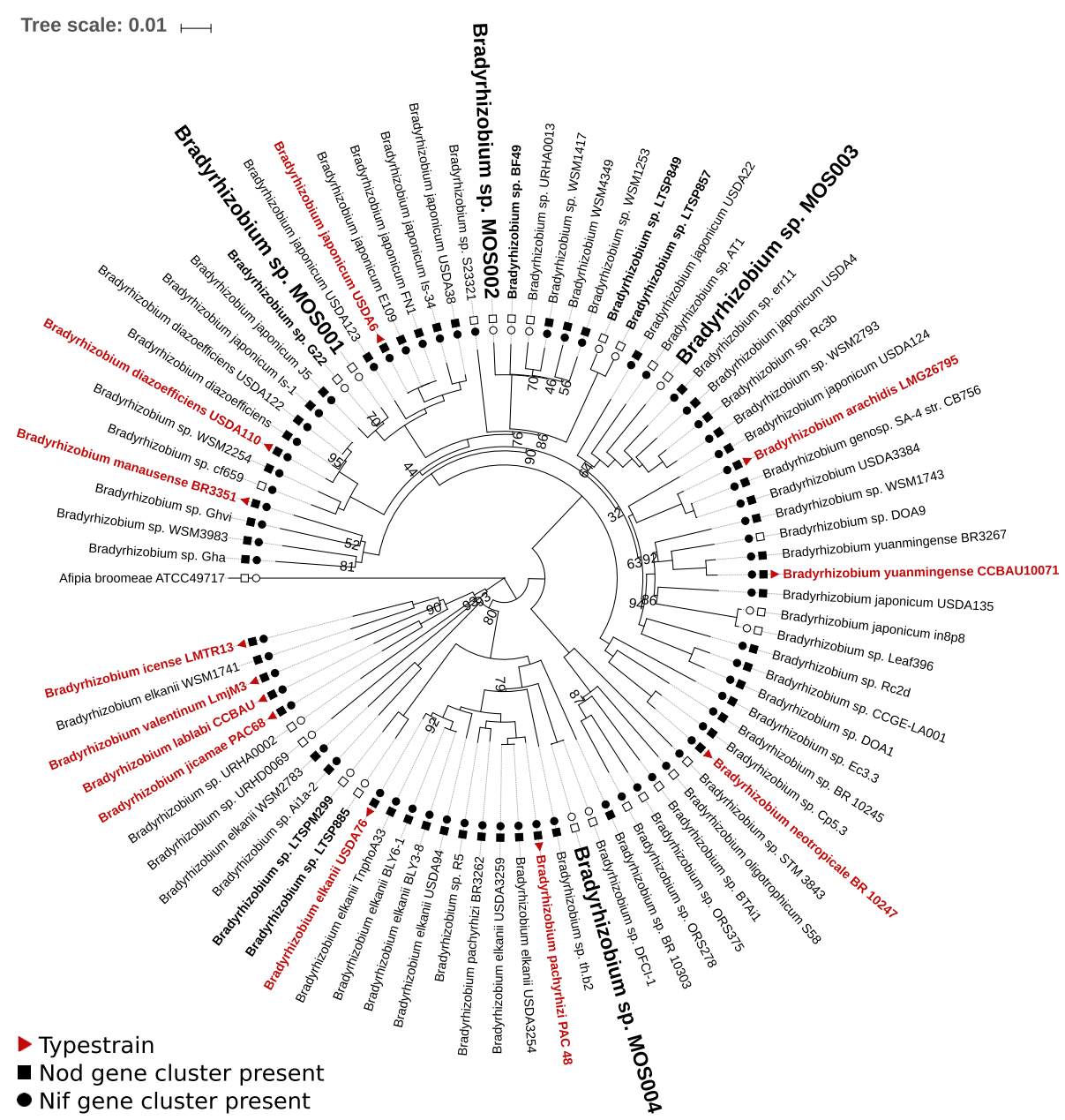

Fig. 3 Phylogenetic analysis shows that the Bradyrhizobium sp. MOS strains are genetically diverse. The tree was constructed using a concatenated nucleotide alignment of 31 AMPHORA genes [18]. For each strain, the presence of the nod/nol gene cluster (filled squares) and the nif/fix gene cluster (filled circles) are indicated. A red triangle means that the particular strain is a type strain for that species [5]. The Bradyrhizobium sp. MOS strains fall into four different clades. Afipia broomae ATCC49717 was used as outgroup

colonized by a diverse range of non-nitrogen fixing Bradyrhizobium species. Earlier studies revealed that Bradyrhizobium species are enriched in rhizosphere soils of non-legumes. For example, in a recent study Bradyrhizobium, but also Rhizobium and Bulkholderia OTUs were found in the core rhizosphere microbiome of a tropical chronosequence comprising 31 plant species [24]. This is in line with a growing body of data that suggests rhizobium-plant interactions are evolutionary conserved and that a non-endosymbiotic interaction may be widespread [12, 13, 24]. Whether the rhizobia identified in those studies possess the symbiotic genes remains to be tested. The fact that the Bradyrhizobium sp. MOS strains that we identified in the root endophytic compartment of Arabidopsis, and other non-legume species, do not have the symbiotic island, shows that the interaction is independent of the known nodulation and fixation genes. Also, we conclude that the colonization of the endophytic compartment by Bradyrhizobium spp. is a common trait and that these Bradyrhizobium spp. most probably have a wide host range.

The four Bradyrhizobium sp. MOS strains were isolated from a single Arabidopsis root. Surprisingly, we found that they are genetically highly diverse. Using 31 conserved amphora genes, we analysed the phylogeny of the MOS strains and all publicly available Bradyrhizobium isolates. Based on analysis by the Average Nucleotide Identity software [25], we conclude that MOS001 represents most probably a $B$. japonicum strain as it shows $\sim 95 \%$ sequence identity with the type strain USDA6. In contrast, MOS002, MOS003 and MOS004 the sequence similarity with known type strains is less than $95 \%$, and therefore these strains possibly represent new species. The fact that the isolates have highly diverse functional profiles suggests that the absence of the symbiotic island is not linked to a specific non-symbiotic 


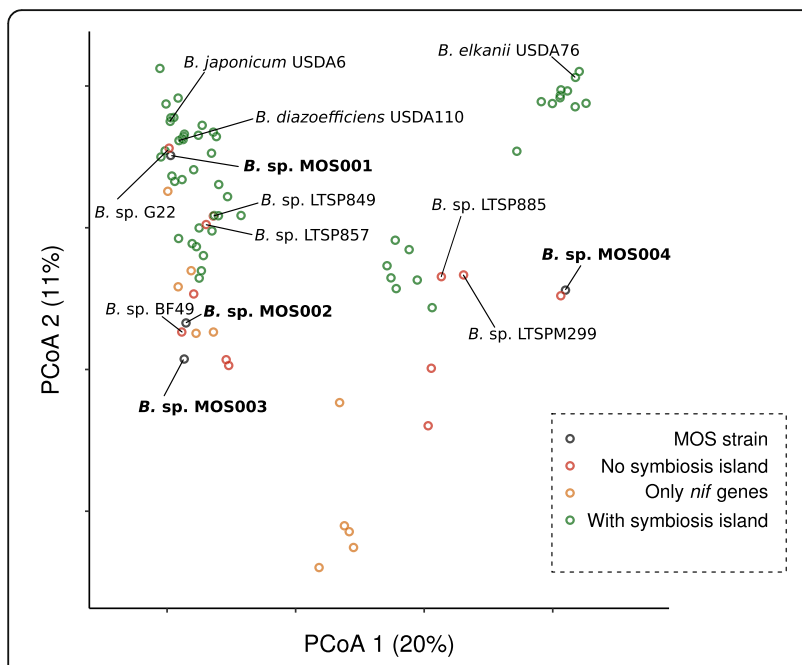

Fig. 4 PCOA on dissimilarities matrix shows that the Bradyrhizobium sp. MOS strains are functionally diverse. Plotted are the first two principal coordinates, which explain $20 \%$ and $11 \%$, respectively. Each circle in the plot represents one functional profile. Green: strains containing the symbiotic island; orange: strains only possessing the nitrogen fixation gene cluster; red: strains lacking the symbiotic island; black: Bradyrhizobium sp. MOS strains, also lacking this island

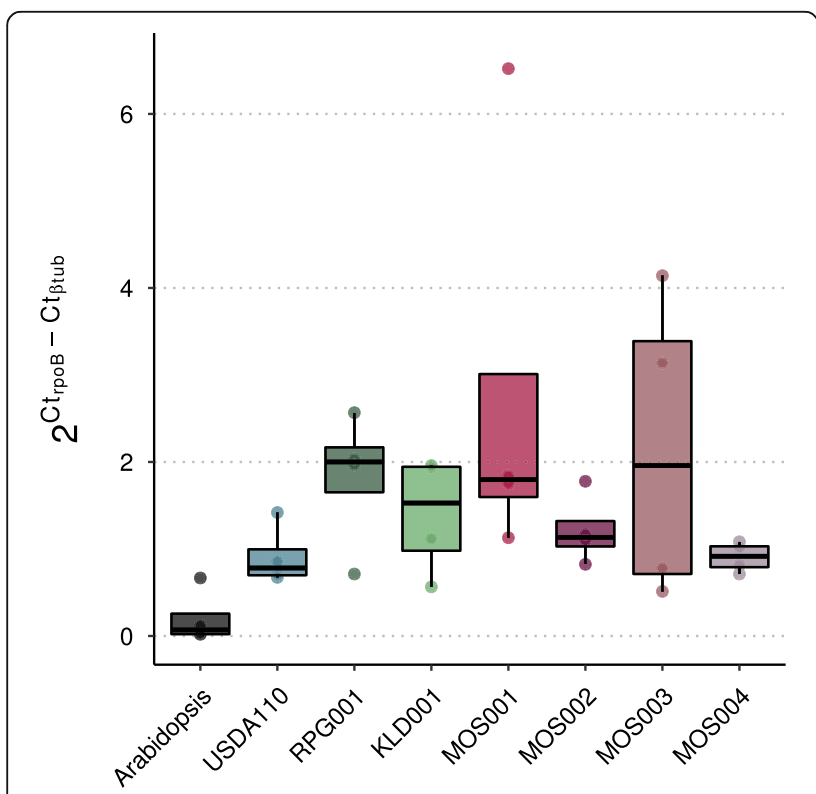

Fig. 5 qPCR-based quantification shows Bradyrhizobium spp. colonization of Arabidopsis roots. On the y-axis an approximation of the number of bacterial genome copies per plant genome copy. This is calculated by exponentiating 2 by the difference between the Ct values of rpoB (targeting bacterial DNA) and Btub (targeting B tubulin in Arabidopsis). Two plant roots grown in autoclaved river sand supplemented with bacteria were pooled, a total of four replicates per treatment was measured. Arabidopsis: mock treated plants. USDA110: Bradyrhizobium diazoefficiens USDA110. RPG001: Bradyrhizobium sp. isolated from Chamaecrista mimosoides nodule. KLD001: Bradyrhizobium sp. isolated from Parasponia andersonii nodule. These strains possess the symbiotic island lifestyle, but that the absence of the symbiotic island is widespread and that these non-diazotrophic species have diverse life strategies.

Re-inoculation experiments on Arabidopsis did not show any plant growth effects of any of our strains under the conditions that we tested. However, it is well possible that within the setting of a complex microbiome, plants as well as the microbes benefit from the close association. We showed that the isolated Bradyrhizobium sp. MOS strains colonize roots with an efficiency comparable to nitrogen-fixing symbionts. In line with this, we argue that an endophytic lifestyle is likely a common trait of Bradyrhizobium sp. Previously, it has been shown that the nodulation and nitrogen fixation genes have been distributed by horizontal gene transfer within the various genera that can establish a nodule symbiosis with legumes $[3,26]$. Therefore it seems probable that Bradyrhizobium strains with an endophytic life style acquired the nod/nol and nif/fix genes by horizontal gene transfer and in this way obtained the specialized nitrogen-fixing symbiotic lifestyle.

\section{Conclusions}

This study shows that Bradyrhizobium spp. colonize the root endophytic compartment of a wide variety of plant species, including the model species Arabidopsis. Four isolates from a single Arabidopsis root have high genetic and functional variation, but all lack the genes for nodule formation and nitrogen fixation. These non-diazotrophic Bradyrhizobium sp. MOS strains, as well as diazotrophic strains, re-colonize the root endophytic compartment of Arabidopsis. Taken together, this study demonstrates that plant roots form a major ecological niche for Bradyrhizobium spp., which might be ancestral to the evolution of the nodulation and nitrogen-fixation trait in this genus.

\section{Methods}

Soil collection and field experiment

Soil was collected in May 2016 at the Mossel area at the 'Hoge Veluwe' in the Netherlands (coordinates: N52 03' $\left.35.5^{\prime \prime} \mathrm{E} 5^{\circ} 45^{\prime} 06.4^{\prime \prime}\right)$, from four different spots within a radius of $100 \mathrm{~m}$. If there was any vegetation present, the top 5-10 $\mathrm{cm}$ soil was removed. The soil was homogenized and all detritus was removed. The soil was kept in a cold room at $4{ }^{\circ} \mathrm{C}$ until use. All plant species were sterilized in $4 \times$ diluted household bleach for $10 \mathrm{~min}$, washed seven times with sterile MQ water and transferred to plates with a wet filter paper, placed at $4{ }^{\circ} \mathrm{C}$ for $48 \mathrm{~h}$ and then moved to a $21{ }^{\circ} \mathrm{C}$ incubator in the dark. Seeds of Arabidopsis Msl also got a short rinse with 70\% ethanol and the seeds of $L$. corniculatus and were treated $2 \mathrm{~min}$ with $\mathrm{H}_{2} \mathrm{SO}_{4}$ before exposure to the household bleach solution. Seeds of T. officinale, T. vulgare, L. vulgare and $M$. arvensis were placed directly in the $21{ }^{\circ} \mathrm{C}$ incubator 
without cold treatment. Mossel soil was placed in a tray with $3 \times 3 \mathrm{~cm}$ pots and watered. To remove the endogenous seed population, the tray was placed in the greenhouse for 2 days. After weeding, the sterile seedlings on the plates were transplanted to the tray with Mossel soil and after 7 days the plants including the soil were planted into to the Mossel field.

\section{Plant harvesting and DNA isolation of the microbial community}

After 6 weeks of growth in the field, plants were excavated using a small shovel $3-4 \mathrm{~cm}$ around the base of the plant. Consequently, the holes were $6-8 \mathrm{~cm}$ wide and around $10 \mathrm{~cm}$ deep. After transporting the plants (including the clod) to the laboratory, we applied the harvesting protocol as described before [27]. Four individual plants were pooled into one sample. DNA was isolated from soil and endophytic compartment samples using the Mo Bio PowerSoil kit (Qiagen) and the Fast DNA Spin Kit for Soil (MP Biomedicals) respectively. Quality and quantity of the DNA was checked by nanodrop and gel electrophoresis. Around $400 \mathrm{ng}$ was sent for $16 \mathrm{~S}$ rDNA sequencing at Beijing Genomics Institute (BGI).

\section{S rDNA amplicon sequencing and data processing}

Using primers 515F and 806R [28], the V4 region was sequenced at BGI on the HiSeq2500 sequencing platform (Illumina). Raw data from BGI was processed using a previously reported custom implementation [29] of QIIME [30] with minor modifications which are described in detail in Schneijderberg et al. (in preparation). In short, reads were quality filtered and filtered for chimeras using ChimeraSlayer. Using a 97\% identity threshold, de novo OTUs were determined, which were taxonomically assigned using the RDP classifier with the GreenGenes database. OTUs related to mitochondrial and chloroplast sequences were removed, as were the OTUs that did not have 25 reads in at least 5 samples. To obtain relative abundance of Bradyrhizobium, reads from OTUs matching Bradyrhizobium were added up per sample, and then divided by the total number of reads of that sample after filtering rare taxa.

\section{Arabidopsis growth assay}

Sterile Arabidopsis Msl Seeds were transferred to $1 / 2 \mathrm{MS}$ plates and incubated at $21{ }^{\circ} \mathrm{C}$ with a $16 \mathrm{~h} / 8 \mathrm{~h}$ photoperiod. After 7 days, roots were ca. $3 \mathrm{~cm}$ in length, and transplanted to pots which were prepared as follows: bacterial cultures were spun down, washed with $10 \mathrm{mM}$ $\mathrm{MgSO}_{4}$ and added to $15 \mathrm{ml}$ of $1 / 4$ Hoagland's medium [31]. This medium was added to $4 \times 4 \mathrm{~cm}$ pots filled with sterile river sand. The soil humidity was set at $70 \%$ of the water holding capacity with a bacterial cell density of $4 \times 10^{5}$ cells per gram of sterile sand. The axenic control was treated with $1 / 4$ Hoagland's only. During the grow period, 5 individual pots were weighed to estimate water loss and if necessary plants were irrigated with sterile water. After 2 weeks, the plants were carefully dug out from the sand and the shoot was separated from the root. Shoot biomass was immediately measured. The roots underwent the harvesting protocol previously described [27] and were stored at $-80{ }^{\circ} \mathrm{C}$ until DNA was extracted as follows: roots were grinded with metal beads in a Tissue Lyzer after which $500 \mu \mathrm{L}$ of CTAB buffer was added. Samples were incubated for $30 \mathrm{~min}$ at $65^{\circ} \mathrm{C}$. Next, $500 \mu \mathrm{L}$ of chloroform was added and the sample was spun down for $5 \mathrm{~min}$ at $14,000 \mathrm{~g}$. Then, the upper phase was transferred to a new Eppendorf tube containing $400 \mu \mathrm{L}$ of isopropanol. The sample was incubated at $-80{ }^{\circ} \mathrm{C}$ for $1 \mathrm{~h}$. After incubation, the mixture was spun down for $10 \mathrm{~min}$ at 14,000 $\mathrm{g}$ and the DNA pellet was washed with $500 \mu \mathrm{L}$ of $70 \%$ ethanol. After another step of centrifugation, the ethanol was removed and the DNA pellet was dried for $15 \mathrm{~min}$ at room temperature. Finally, the pellet was resuspended in $50 \mu \mathrm{L}$ of MilliQ water and stored at $-2{ }^{\circ} \mathrm{C}$ until further use.

\section{Quantitative PCR}

Primers targeting the rpoB gene in Bradyrhizobium spp. (rpoB_Fw1; 5'-CGCTGAAGAACCTCGACGAAGCC-3' and rpoB_Rv1; 5'-CGGCGTGATCTTGCCGACCAG-3') were designed using Geneious 8.1. The Arabidopsis Btubulin gene primers (Forward: 5'-AGAAAACCGGA AACGAGAGC-3' and Reverse 5'-ACAAGACACTTT CCGCTTGG-3') were used to normalize against plant DNA [23]. Both primer sets were tested for efficiency prior to the qPCR. The qPCR was performed on four plant DNA samples per treatment, by following the manufacturer's protocol (Power SYBR Green, Life Technologies). To determine bacterial versus plant DNA, we used the formula:

$$
Y=2^{(C t(r p o B)-C t(t u b))}
$$

which is an approximation of the number of bacterial genome copies versus plant genome copy number.

\section{Strain isolation and culturing}

Sonicated and cleaned Arabidopsis root tissue was ground with mortar and pestle in $1 \mathrm{ml}$ phosphate buffer (per litre: $6.33 \mathrm{~g}$ of $\mathrm{NaH}_{2} \mathrm{PO}_{4} \cdot \mathrm{H}_{2} \mathrm{O}, 10.96 \mathrm{~g}$ of $\mathrm{Na}_{2} \mathrm{HPO}_{4} \cdot 2 \mathrm{H}_{2} \mathrm{O}$ and $200 \mu \mathrm{L}$ Silwet L-77). $100 \mu \mathrm{L}$ of the resulting solution was plated in duplicate on $1 / 10$ TSA and YEM medium plates. $500 \mu \mathrm{L}$ of the remaining solution was stored in $40 \%$ glycerol for later use. In addition, a $100 \times$ and $1000 \times$ fold dilution were also plated in duplicate. Colonies appearing after 
3 days and 7 days were picked (Additional file 2), and stored in YEM medium in 96 well format.

\section{Genomic DNA isolation for sequencing of the bacterial strains}

Strains were inoculated in $10 \mathrm{ml}$ liquid YEM medium. After 5 days of growth, cells were centrifuged at $4000 \mathrm{~g}$, and the medium was discarded. For isolation of DNA, the Qiagen Blood \& Tissue kit was used, according to the manufacturer's instructions. DNA quantity and purity was checked by nanodrop and gel electrophoresis. MOS003 underwent one more round of DNA isolation because the total quantity was not enough to meet the minimal requirements for sequencing.

\section{Sequencing of the bacterial strains}

Sequencing was performed at BGI, using the Illumina HiSeq 2500 PE 150 platform, with a 350 bp insert library size. Paired-end Illumina reads were quality assessed with FastQC [32], and trimmed accordingly with Trimmomatic v. 0.35 [33], and all samples were filtered for a PHRED33 threshold score above 20. Raw reads were assembled with SPAdes v. 3.9.0 [34] under different k-mer sizes with the best assembly judged as containing the smallest number of contigs. Contigs < 1000 bp (only present in MOS002) were removed from the genomes. Assembly statistics were evaluated with QUAST v. 4.5 [35]. The GC content of the contigs revealed contamination in MOS001 and MOS004. The contamination was removed with MaxBin v. 2.2.4 [36]. The ORFs and proteome of the Mossel assemblies were predicted with Prodigal v. 2.6.1 [37]. Assemblies were annotated with the Prokka v1.11 tool [38]. A local BLAST database was made from RefSeq proteins belonging to the Bradyrhizobium genus with the BLAST+ package [39]. By specifying the genus flag, Prokka first annotated genes with respect to this database.

\section{Symbiotic island homology}

All publicly available draft and complete assemblies belonging to the Bradyrhizobium genus were pulled from NCBI in January 2017. Symbiotic genes were identified with a BLAST reciprocal best hit algorithm [39]. The genes on the symbiotic island of the $B$. diazoefficiens USDA110 assembly was used as the reference for orthologue searching. Presence or absence was defined as a putative orthologue with a minimum $70 \%$ identity and $50 \%$ coverage as compared to the reference gene.

\section{Phylogenetic diversity analysis}

The 31 AMPHORA genes were found to be present in all the assemblies. The nucleotide sequence of inidividal AMPHORA genes were aligned with Clustal Omega v. 1.2.4 [40] and non-conserved segments were removed with Gblocks [41]. Using the neighbour-joining method in Geneious 8.1, the phylogenetic tree was generated with 1000 bootstraps. The tree was visualized by using Interactive Tree of Life (iTol) platform [42].

\section{Analyses of functional diversity}

The presence or absence of KEGG Orthologue (KO) groups was determined in the ORFs by employing HMM models publically published by Bai et al. [21]. The KO groups were identified using the HMMER v. 3.1b2 [43] hmmsearch tool with thresholds set at an E-value smaller than $10 \times 10^{-5}$ and larger than $70 \%$ coverage. For multiple hits meeting this criteria, only the hit with the smallest Evalue was retained. The data was transformed from absolute counts per $\mathrm{KO}$ group to either absent or present. Using the vegdist command from the Vegan package in $\mathrm{R}$, a distance matrix was created using the binary distance measure. PcoA was performed by the $c m d s c a l e$ command of the Stats package in $\mathrm{R}$, with Bray-Curtis as method for dissimilarity.

\section{Additional files}

Additional file 1: Table S1. DNA fingerprinting of 102 isolates (XLSX $12 \mathrm{~kb}$ ) Additional file 2: Table S2. Assembly statistics (XLSX $11 \mathrm{~kb}$ )

Additional file 3: Figure S1. Bradyrhizobium spp. do not affect Arabidopsis shoot fresh weight. Plants were grown for 14 days on sterilised river sand, supplemented with the Bradyrhizobium spp. strains, or mock treated (Control). Only shoot weight was measured, roots were used as template for the GPCR in Fig. 5. Each dot represents one replicate ( $n=30$ for each treatment, except for RPG001, for which is $n=29$ ). (PDF $6 \mathrm{~kb}$ )

\section{Acknowledgments}

We would like to thank the students of the course Molecular Toolbox \#60 and \#63 at the Wageningen University for executing pilot experiments, Dr. Marnix Medema for his valuable comments on the bioinformatics and Yosapol Harnvanichvech and Zhichun Yan for their help during preparation of this manuscript.

\section{Funding}

This research was funded by ERC grant number 3100000843 .

\section{Availability of data and materials}

The Bradyrhizobium sp. MOS draft genomes are available on the NCBI under accession numbers SAMN07975002 (MOS001) and SAMN08687197 SAMN08687199 (MOS002 - MOS004). The 165 amplicon data is part of a manuscript in preparation. A draft version of that manuscript and the corresponding 165 amplicon data is available upon request.

\section{Authors' contributions}

MS, SP, XC and CF isolated and characterised the bacterial strains. MS and LS assembled the genomes and executed in silico analyses. MS, XC and CF executed other experiments. MS, RG and TB wrote the manuscript. All authors read and approved the final manuscript.

Ethics approval and consent to participate Not applicable.

Consent for publication

Not applicable. 


\section{Competing interests}

The authors declare that they have no competing interests.

\section{Publisher's Note}

Springer Nature remains neutral with regard to jurisdictional claims in published maps and institutional affiliations.

\section{Received: 3 December 2017 Accepted: 26 March 2018}

\section{Published online: 11 April 2018}

\section{References}

1. Masson-Boivin C, Giraud E, Perret X, Batut J. Establishing nitrogen-fixing symbiosis with legumes: how many rhizobium recipes? Trends Microbiol. 2009;17:458-66.

2. Udvardi M, Poole PS. Transport and metabolism in legume-rhizobia symbioses. Annu Rev Plant Biol. 2013;64:781-805.

3. Remigi P, Zhu J, Young JPW, Masson-Boivin C. Symbiosis within symbiosis: evolving nitrogen-fixing legume symbionts. Trends Microbiol. 2016;24:63-75.

4. Jordan DC. NOTES: transfer of rhizobium japonicum Buchanan 1980 to Bradyrhizobium gen. Nov., a genus of slow-growing, root nodule Bacteria from leguminous plants. Int J Syst Bacteriol. 1982;32:136-9.

5. Parte AC. LPSN - list of prokaryotic names with standing in nomenclature. Nucleic Acids Res. 2014;42:D613-6.

6. Trinick MJ, Hadobas PA. Biology of the Parasponia-Bradyrhizobium symbiosis. Plant Soil. 1988;110:177-85.

7. van Rhijn P, Vanderleyden J. The rhizobium-plant symbiosis. Microbiol Rev. 1995:59:124-42

8. Batut J, Andersson SGE, O'Callaghan D. The evolution of chronic infection strategies in the a -proteobacteria. Nat Rev Microbiol. 2004;2:933-45.

9. Sachs $J$, Ehinger $M O$, Simms EL. Origins of cheating and loss of symbiosis in wild Bradyrhizobium. J Evol Biol. 2010;23:1075-89.

10. Hartmann M, Howes CG, Vaninsberghe D, Yu H, Bachar D, Christen R, et al. Significant and persistent impact of timber harvesting on soil microbial communities in northern coniferous forests. ISME J. 2012;6:2199-218.

11. Uroz S, Buée M, Murat C, Frey-Klett P, Martin F. Pyrosequencing reveals a contrasted bacterial diversity between oak rhizosphere and surrounding soil. Environ Microbiol Rep. 2010;2:281-8.

12. Vanlnsberghe D, Maas KR, Cardenas E, Strachan CR, Hallam SJ, Mohn WW. Non-symbiotic Bradyrhizobium ecotypes dominate north American forest soils. ISME J. 2015;9:2435-41.

13. Jones FP, Clark IM, King R, Shaw $\sqcup$, Woodward MJ, Hirsch PR. Novel European free-living, non-diazotrophic Bradyrhizobium isolates from contrasting soils that lack nodulation and nitrogen fixation genes - a genome comparison. Sci Rep. 2016:6:25858.

14. Wang Q, Garrity GM, Tiedje JM, Cole JR. Naïve Bayesian classifier for rapid assignment of rRNA sequences into the new bacterial taxonomy. App Environ Microbiol. 2007;73:5261-7.

15. Kaneko T, Nakamura Y, Sato S, Minamisawa K, Uchiumi T, Sasamoto S, et al. Complete genomic sequence of nitrogen-fixing symbiotic bacterium Bradyrhizobium japonicum USDA110. DNA Res. 2002;9:189-97.

16. Sugawara M, Tsukui T, Kaneko T, Ohtsubo Y, Sato S, Nagata Y, et al. Complete genome sequence of Bradyrhizobium diazoefficiens USDA 122, a nitrogen-fixing soybean symbiont. Genome Announc. 2017;5:e01743-16.

17. Simão FA, Waterhouse RM, loannidis $P$, Kriventseva EV, Zdobnov EM BUSCO: assessing genome assembly and annotation completeness with single-copy orthologs. Bioinformatics. 2015;31:3210-2.

18. Wu M, Eisen JA. A simple, fast, and accurate method of phylogenomic inference. Genome Biol. 2008:9:R151.

19. Rivas R, Martens M, de Lajudie P. Willems A. Multilocus sequence analysis of the genus Bradyrhizobium. Syst Appl Microbiol. 2009;32:101-10.

20. Zhang XX, Guo HJ, Wang R, Sui XH, Zhang YM, Wang ET, et al. Genetic divergence of Bradyrhizobium strains nodulating soybeans as revealed by multilocus sequence analysis of genes inside and outside the symbiosis island. Appl Environ Microbiol. 2014:80:3181-90.

21. Bai $Y$, Müller DB, Srinivas G, Garrido-Oter R, Potthoff E, Rott M, et al. Functional overlap of the Arabidopsis leaf and root microbiota. Nature 2015;528:364-9.

22. Ogata H, Goto S, Sato K, Fujibuchi W, Bono H, Kanehisa M. KEGG: Kyoto encyclopedia of genes and genomes. Nucleic Acids Res. 1999;27:29-34.

23. Li H, Torres-Garcia J, Latrasse D, Benhamed M, Schilderink S, Zhou W, et al. Plant-specific histone deacetylases HDT $1 \frac{1}{2}$ regulate GIBBERELLIN 2-OXIDASE
2 expression to control Arabidopsis root meristem cell number. Plant cell. 2017:29:tpc. 2017:00366.

24. Yeoh YK, Dennis PG, Paungfoo-Lonhienne C, Weber L, Brackin R, Ragan MA, et al. Evolutionary conservation of a core root microbiome across plant phyla along a tropical soil chronosequence. Nat Commun. 2017;8:215.

25. Yoon SH, Ha S. Min, Lim J, kwon S, Chun J. A large-scale evaluation of algorithms to calculate average nucleotide identity. Antonie van Leeuwenhoek, Int J gen. Mol Microbiol. 2017;110:1281-6.

26. Sachs JL, Skophammer RG, Regus JU. Evolutionary transitions in bacterial symbiosis. Proc Natl Acad Sci. 2011;108(Supplement 2):10800-7.

27. Lundberg DS, Lebeis SL, Paredes SH, Yourstone S, Gehring J, Malfatti S, et al. Defining the core Arabidopsis thaliana root microbiome. Nature. 2012;488: 86-90.

28. Parada AE, Needham DM, Fuhrman JA. Every base matters: assessing smal subunit rRNA primers for marine microbiomes with mock communities, time series and global field samples. Environ Microbiol. 2016;18:1403-14.

29. Pérez-Jaramillo JE, Carrión VJ, Bosse M, Ferrão LFV, De Hollander M, Garcia $A A F$, et al. Linking rhizosphere microbiome composition of wild and domesticated Phaseolus vulgaris to genotypic and root phenotypic traits. ISME J. 2017:11:2244-57.

30. Caporaso JG, Kuczynski J, Stombaugh J, Bittinger K, Bushman FD, Costello EK, et al. QIIME allows analysis of high-throughput community sequencing data. Nat Methods. 2010;7:335-6.

31. Hoagland DR, Arnon DI. The water-culture method for growing plants without soil. Calif Agric Exp Stn Circ. 1950;347:1-32.

32. Andews S. FastQC: a quality control tool for high throughput sequence data. https://www.bioinformatics.babraham.ac.uk/projects/fastqc/.

33. Bolger AM, Lohse M, Usadel B. Trimmomatic: a flexible trimmer for Illumina sequence data. Bioinformatics. 2014;30:2114-20.

34. Bankevich A, Nurk S, Antipov D, Gurevich AA, Dvorkin M, Kulikov AS, et al. SPAdes: a new genome assembly algorithm and its applications to singlecell sequencing. J Comput Biol. 2012;19:455-77.

35. Gurevich A, Saveliev V, Vyahhi N, Tesler G. QUAST: quality assessment tool for genome assemblies. Bioinformatics. 2013;29:1072-5.

36. Wu Y-W, Tang Y-H, Tringe SG, Simmons BA, Singer SW. MaxBin: an automated binning method to recover individual genomes from metagenomes using an expectation-maximization algorithm. Microbiome. 2014;2:26.

37. Hyatt D, Chen G-L, LoCascio PF, Land ML, Larimer FW, Hauser LJ. Prodigal: prokaryotic gene recognition and translation initiation site identification. BMC Bioinformatics. 2010;11:119.

38. Seemann T. Prokka: rapid prokaryotic genome annotation. Bioinformatics. 2014;30:2068-9.

39. Cock PJA, Chilton JM, Grüning B, Johnson JE, Soranzo N. NCBI BLAST+ integrated into galaxy. Gigascience. 2015;4:39.

40. Sievers F, Wilm A, Dineen D, Gibson TJ, Karplus K, Li W, et al. Fast, scalable generation of high-quality protein multiple sequence alignments using Clustal omega. Mol Syst Biol. 2011:7:539.

41. Castresana J. Selection of conserved blocks from multiple alignments for their use in phylogenetic analysis. Mol Biol Evol. 2000;17:540-552.

42. Letunic I, Bork P. Interactive tree of life (iTOL) v3: an online tool for the display and annotation of phylogenetic and other trees. Nucleic Acids Res. 2016:44:W242-5.

43. Eddy SR. Profile hidden Markov models. Bioinformatics. 1998;14:755-63.

44. Bulgarelli D, Rott M, Schlaeppi K. Ver Loren van Themaat E, Ahmadinejad N, Assenza $\mathrm{F}$, et al. revealing structure and assembly cues for Arabidopsis rootinhabiting bacterial microbiota. Nature. 2012:488:91-5.

45. Lebeis SL, Paredes SH, Lundberg DS, Breakfield N, Gehring J, McDonald M, et al. Salicylic acid modulates colonization of the root microbiome by specific bacterial taxa. Science. 2015;349:860-4.

46. Edwards J, Johnson C, Santos-Medellín C, Lurie E, Podishetty NK, Bhatnagar $\mathrm{S}$, et al. Structure, variation, and assembly of the root-associated microbiomes of rice. Proc Natl Acad Sci. 2015;112:E911-20.

47. Niu B, Paulson JN, Zheng X, Kolter R. Simplified and representative bacterial community of maize roots. Proc Natl Acad Sci. 2017;114:E2450-9. 